\title{
Une méthode d'homogénéisation pour l'analyse modale d'un réacteur nucléaire avec modélisation des structures internes et de l'interaction fluide/structure
}

\author{
JeAn-FranÇOis Sigrist ${ }^{1, a}$ ET DANiel Broc ${ }^{2}$ \\ 1 DCN Propulsion, Service Technique et Scientifique, 44620 La Montagne, France \\ 2 CEA Saclay, Laboratoire d'Étude Mécanique et Sismique, 91191 Gif-sur-Yvette, France
}

Reçu le $1^{\text {er }}$ septembre 2006, accepté le 23 janvier 2007

\begin{abstract}
Résumé - Les méthodes d'homogénéisation ont été utilisées de façon assez courante dans l'industrie nucléaire, en particulier pour la modélisation des faisceaux de tubes. L'application de cette approche à la modélisation numérique d'un réacteur nucléaire, avec prise en compte des effets de couplage et de confinement induits par la présence des structures internes du réacteur, est étudiée dans le présent article. La modélisation est ainsi basée sur une approche homogénéisée de l'interaction fluide/structure entre les structures internes, réparties de façon périodique dans le réacteur, et le fluide contenu entre la structure constituant l'enveloppe résistante et la structure de supportage du cœur. Les bases théoriques de l'approche homogénéisée sont rappelées dans un premier temps et la méthode est ensuite détaillée dans le cas de structures rigides périodiques. La validation numérique de l'approche couplée est établie par comparaison entre une modélisation tridimensionnelle «complète » du réacteur avec détail de la géométrie des structures internes et des espaces fluides et une modélisation bidimensionnelle « réduite » utilisant un fluide homogène équivalent. L'importance des effets de confinement est ainsi mise en évidence dans le cas industriel étudié.
\end{abstract}

Mots clés : Interaction fluide / structure / analyse modale / méthode d'homogénéisation / application industrielle

\begin{abstract}
A homogenisation method for the modal analysis of a nuclear reactor with internal structures modelling and fluid-structure interaction coupling. A homogenisation method is presented and validated in order to perform the dynamic analysis of a nuclear pressure vessel with a "reduced" numerical model accounting for inertial fluid-structure coupling and describing the geometrical details of the internal structures, periodically embedded within the nuclear reactor. Homogenisation techniques have been widely used in nuclear engineering to model confinement effects in reactor cores or tubes bundles. Application of such techniques to reactor internals is investigated in the present paper. The theory bases of the method are first recalled. Adaptation of the homogenisation approach to the case of reactor internals is then exposed: it is shown that in such case, confinement effects can be modelled by a suitable modification of classical fluid-structure symmetric formulation. The method is then validated by comparison of 3D and 2D calculations. In the latter, a "reduced" model with homogenised fluid is used, whereas in the former, a full finite element model of the nuclear pressure vessel with internal structures is elaborated. The homogenisation approach is proved to be efficient from the numerical of view point and accurate from the physical point of view. Confinement effects in the industrial case can then be highlighted.
\end{abstract}

Key words: Fluid-structure interaction / modal analysis / homogenisation method / industrial application

\section{Introduction}

Les effets d'interaction fluide/structure se produisent à des degrés divers pour toute structure en contact avec

\footnotetext{
${ }^{a}$ Auteur correspondant : jean-francois.sigrist@dcn.fr
}

un fluide au repos [1]. Les phénomènes de couplage fluide/structure ont fait l'objet d'études importantes au cours des dernières années, sur les plans expérimental, théorique ou numérique [2-5]. L'analyse des effets d'interaction fluide/structure doit cependant être conduite pour 


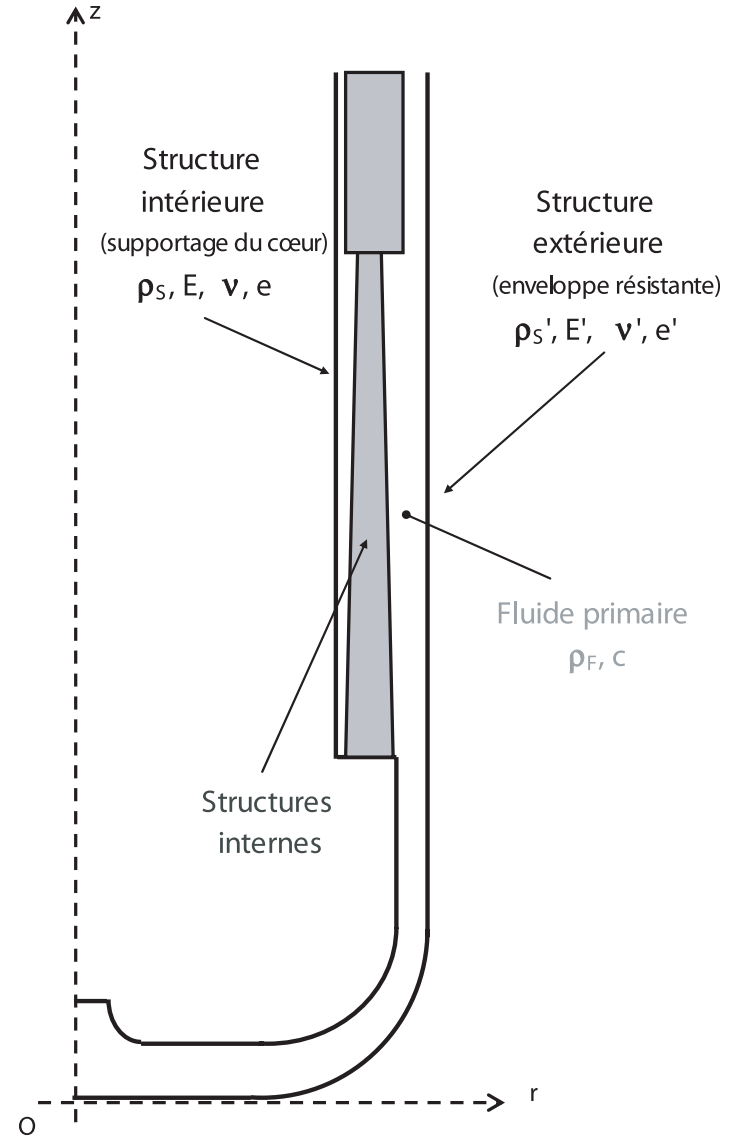

Fig. 1. Schématisation d'un réacteur de propulsion navale.

chaque application industrielle, puisque l'influence de la présence du fluide peut être différente d'un cas à un autre.

En tant que constructeur de réacteurs nucléaires de propulsion navale (prototypes à terre ou réacteurs embarqués), DCN Propulsion a mis en place au cours des dernières années un programme d'étude amont visant à prendre en compte les effets d'interaction fluide/structure dans les études de dimensionnement des équipements de propulsion $[6,7]$. Ainsi, l'influence des effets d'interaction sur le comportement dynamique de réacteurs a été analysée dans des études précédentes pour la gamme de fréquence couvrant le spectre du chargement appliqué sur les équipements.

Les analyses réalisées ne prenaient cependant pas en compte la présence des structures internes du réacteur (voir Fig. 1) dont l'effet est d'accroître le confinement du fluide primaire contenu dans le réacteur. L'objet de la présente étude est d'évaluer l'importance de cet effet de confinement et de proposer une modélisation des structures internes et du couplage fluide/structure.

En première approximation, le couplage fluide/ structure peut être décrit en ne prenant pas en compte la présence des structures internes : l'espace annulaire compris entre l'enveloppe du réacteur et la structure de supportage du cœur est ainsi supposé rempli d'un fluide sous pression, de masse volumique donnée (ce qui pour une section de cote donnée correspond au cas schématisé par

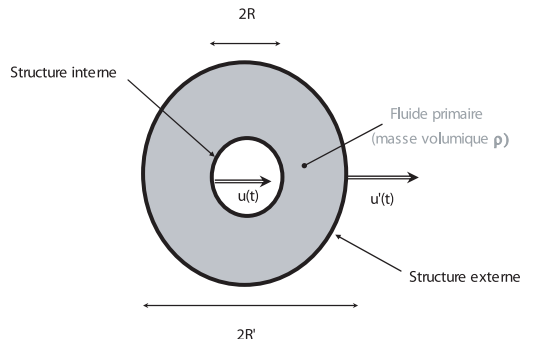

(a)

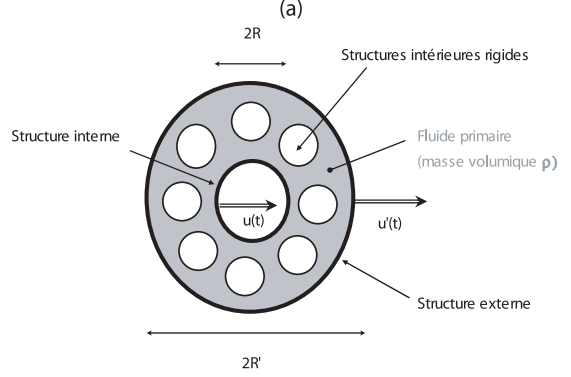

(b)

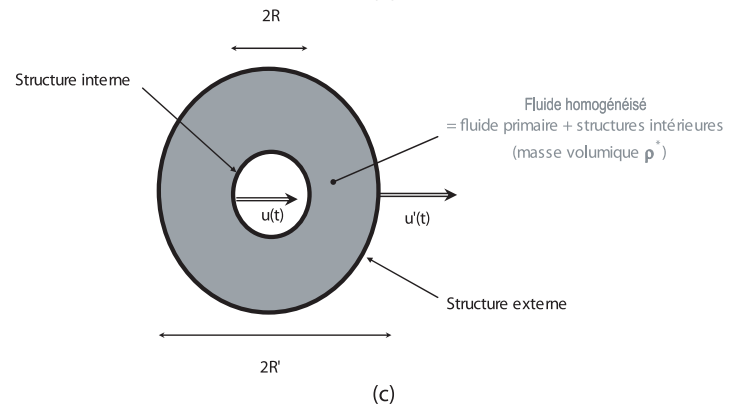

Fig. 2. Modèle par tranche avec fluide, avec ou sans structures internes et modèle fluide homogénéisé équivalent.

la Fig. 2a). Cette modélisation permet ainsi de prendre en compte les effets de couplage inertiels entre les deux structures couplées par le fluide. Le formalisme mathématique de l'interaction est alors bien établi [3] et de nombreux codes de calcul permettent de réaliser l'analyse en utilisant un couplage éléments-finis/éléments-finis [8].

Dans une approche plus générale, la description des effets de couplage fluide/structure avec prise en compte des structures intérieures peut se faire :

- soit en utilisant une modélisation détaillée de la géométrie des inclusions solides et de leur répartition dans le réacteur. Dans ce cas, les effets inertiels et de confinement du fluide sont explicitement décrits par la discrétisation du problème, qui requiert néanmoins un maillage complexe de la géométrie tridimensionnelle du réacteur et de ses structures intérieures. Cette approche correspond au cas schématisé par la figure $2 \mathrm{~b}$;

- soit en utilisant une modélisation du fluide et des inclusions solides, sous forme d'un domaine homogénéisé équivalent. Dans ce cas, les effets de couplage fluide/structure sont décrits de façon implicite, qui ne nécessite pas de maillage complexe de l'espace intérieur du réacteur, ce qui correspond schématiquement à la situation décrite par la figure 2c. Cette approche est basée sur l'utilisation de méthodes 
d'homogénéisation, dont la pratique est rendue ici pertinente par le caractère périodique de la géométrie du problème.

Les méthodes d'homogénéisation ont été développées et appliquées dans de nombreux domaines, par exemple pour des structures présentant des périodicités géométriques [9] ou pour des fluides en milieux poreux [10]. Ces méthodes ont également été appliquées aux problèmes d'interaction fluide/structure [11], en particulier pour des applications dans le domaine nucléaire, comme la modélisation des faisceaux de tubes [12], des échangeurs de chaleur [13,14] ou des cours de réacteur $[15,16]$.

Le présent article expose une application de l'approche homogénéisée à un problème d'interaction fluide/structure au cas de la modélisation des structures internes ${ }^{1}$ d'un réacteur nucléaire. Ce cas d'étude diffère des exemples cités précédemment au moins à deux titres :

- le nombre d'inclusions solides est, dans le cas des internes du réacteur de propulsion navale, nettement inférieur à celui du faisceau de tubes (plusieurs dizaines dans le premier cas, plusieurs milliers dans le second cas) ;

- la géométrie du problème est ici caractérisée par un confinement type «cylindre dans cylindre », alors que le confinement dans les cours de réacteurs est du type « carré dans carré »

Le besoin industriel final est de conduire une analyse dynamique du comportement d'ensemble du réacteur sous sollicitation type sismique ou choc, au moyen de méthodes modales [17]. Ces méthodes requièrent ainsi une analyse modale complète du système (calcul des déformées, fréquences et masses modales) avec prise en compte des effets d'interaction fluide/structure. Ce point conditionne la mise en œuvre de l'analyse dynamique et la méthode proposée a pour objectif une application industrielle de l'approche homogénéisée, en particulier en la rendant compatible avec la formulation couplée symétrique $(\boldsymbol{u}, p, \varphi) \mathrm{du}$ problème couplé [3].

La méthode proposée est dérivée de l'approche générale proposée par Hammami et Brochard pour l'analyse sismique d'un faisceau de tubes [18]. La méthode est ici adaptée au cas des internes du réacteur, supposées rigides et solidaires de la structure de supportage du cœur, sans introduire ainsi de degré de liberté additionnel pour le problème fluide, ce qui permet une implantation directe dans un code de calcul éléments-finis, en modifiant des opérateurs de couplage déjà existants.

\footnotetext{
1 Dans la suite de l'article, on utilisera le vocable « internes » consacré par l'usage pour désigner les structures internes du réacteur.
}

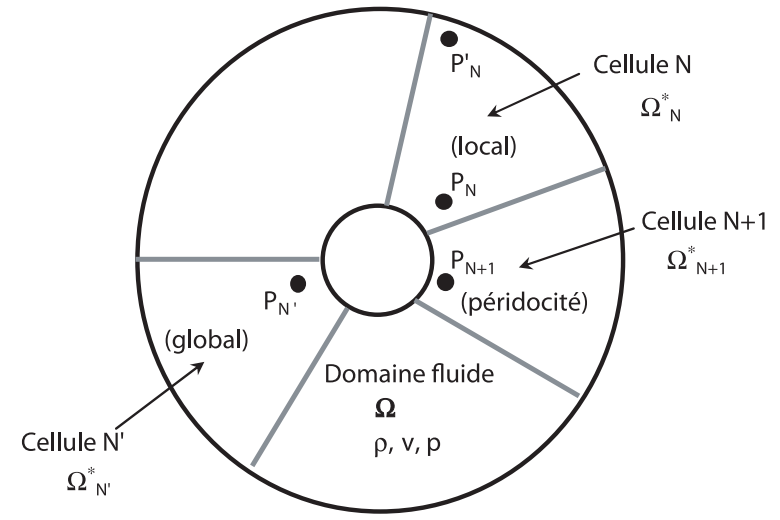

Fig. 3. Domaine périodique $\Omega$ divisé en cellules élémentaires $\Omega_{\mathrm{N}}$.

\section{Méthode d'homogénéisation pour un problème d'interaction fluide/structure}

\subsection{Principe de base de la méthode d'homogéné- isation pour un problème couplé fluide/structure périodique}

Pour un problème couplé fluide/structure qui présente une périodicité spatiale, l'approche homogénéisée consiste à diviser le domaine étudié en cellules élémentaires (voir par exemple la figure 3 dans le cas de deux cylindres concentriques) avec une période en espace caractéristique, supposée « petite » devant une grandeur caractéristique de la géométrie d'ensemble. On peut alors définir un paramètre $\varepsilon$ caractérisant la périodicité du problème.

Par suite, les inconnues du problème (champ de pression $p$ et de vitesse $\boldsymbol{v}$ dans le fluide et champ de déplacement de la structure $\boldsymbol{u}$ ) peuvent être vues comme des fonctions dépendantes de $\varepsilon$, pour lesquelles on écrit un développement asymptotique en double échelle, de la forme :

$$
\begin{gathered}
p_{\varepsilon}=p_{\mathrm{o}}+\sum_{k \geqslant 1} \varepsilon^{k} p_{k}(X, x) \\
\boldsymbol{v}_{\varepsilon}=\boldsymbol{v}_{\mathrm{O}}+\sum_{k \geqslant 1} \varepsilon^{k} \boldsymbol{v}_{k}(X, x) \\
\boldsymbol{u}_{\varepsilon}=\boldsymbol{u}_{\mathrm{o}}+\sum_{k \geqslant 1} \varepsilon^{k} \boldsymbol{u}_{k}(X, x)
\end{gathered}
$$

où les fonctions $p_{k}, \boldsymbol{v}_{k}$ et $\boldsymbol{u}_{k}$ dépendent d'une « variable locale $»$ notée $x$ et d'une «variable globale $»$ notée $X$ en posant $x=X / \varepsilon$.

Les fonctions $p_{k}, \boldsymbol{v}_{k}$ et $\boldsymbol{u}_{k}$ sont d'une part « pseudo périodiques » par rapport à la variable globale, ce qui traduit le fait qu'elles prennent des valeurs identiques pour deux points équivalents dans des cellules voisines (par exemple aux points $P_{N}$ et $P_{N+1}$, voir Fig. 3 ) et qu'elles prennent des valeurs quasi identiques en deux points équivalents pour deux cellules distantes dans le domaine (par exemple aux points $P_{N}$ et $P_{N^{\prime}}$, Fig. 3). D'autre part, $p_{k}, \boldsymbol{v}_{k}$ et $\boldsymbol{u}_{k}$ dépendent de la variable d'échelle locale 
au sens où elles prennent des valeurs différentes en deux points distincts d'une même cellule (points $P_{N}$ et $P_{N}^{\prime}$, Fig. 3). La technique d'homogénéisation consiste ainsi à déduire une description globale du problème périodique fluide/structure (au niveau du domaine homogénéisé) à partir de la description locale (au niveau de la cellule élémentaire).

En substituant les expressions (1) à (3) dans les équations décrivant la dynamique vibratoire de la structure en contact avec un fluide supposé non visqueux, initialement au repos (de masse volumique $\rho_{\mathrm{F}}$ ) et en identifiant des termes de même puissance en $\varepsilon$, on déduit alors les équations suivantes [18] :

$$
\begin{gathered}
\frac{\partial \tilde{\rho}_{\mathrm{o}}}{\partial t}+\rho_{\mathrm{F}} \frac{\partial \tilde{v}_{\mathrm{oi}}}{\partial X_{\mathrm{i}}}=0 \\
\bar{\rho} \frac{\partial \tilde{v}_{\mathrm{oi}}}{\partial t}=-(1-J) \frac{\partial p_{\mathrm{o}}}{\partial X_{\mathrm{i}}}+\rho_{\mathrm{F}} J \frac{\partial^{2} u_{\mathrm{oi}}}{\partial t^{2}}
\end{gathered}
$$

où $\tilde{\boldsymbol{v}}$ et $\tilde{\rho}$ représentent les valeurs moyennes sur une cellule fluide des fluctuations de vitesse et de masse volumique autour de l'état initial au repos. $J$ est le ratio de confinement, défini comme :

$$
J=1-\frac{1}{\left|\Omega^{*}\right|} \int_{\Omega^{*}}\left(1-\frac{\partial \Pi_{\mathrm{i}}}{\partial x_{\mathrm{i}}}\right) \mathrm{d} \Omega^{*}
$$

où $\Pi_{\mathrm{i}}$ est solution du problème élémentaire $\Delta \Pi_{\mathrm{i}}=0$ dans $\Omega^{*}$ avec la condition aux limites $\frac{\partial \Pi_{\mathrm{i}}}{\partial n}=\boldsymbol{e}_{\mathrm{i}} \cdot \boldsymbol{n}$ sur $\partial \Omega^{*}$ et la contrainte $\int_{\Omega^{*}} \Pi_{\mathrm{i}} \mathrm{d} \Omega^{*}=0\left(\Omega^{*}\right.$ désigne ici une cellule élémentaire fluide et $\boldsymbol{e}_{\mathrm{i}}$ est un vecteur unité dans la direction $i$ ). Les équations (4) et (5) décrivent la conservation de la masse et de la quantité de mouvement du point de vue du fluide homogénéisé et prennent en compte les effets de couplage avec les structures en mouvement dans la cellule. Ces équations peuvent ensuite être spécialisées au cas des internes du réacteur [19], pour lequel les inclusions solides sont supposées rigides et liées à la coque interne.

\subsection{Application de l'approche homogénéisée au cas d'inclusions solides rigides}

Dans le cas du réacteur nucléaire, les dimensions des structures extérieures et intérieures et le nombre de structures internes sont tels qu'une cellule fluide élémentaire peut être représentée comme suit, figure 4.

Sur chaque cellule, on note $\Omega_{\mathrm{S}}$ le domaine occupé par l'inclusion solide, $\Omega_{\mathrm{F}}$ le domaine occupé par le fluide et $\Omega_{\mathrm{T}}=\Omega_{\mathrm{S}} \cup \Omega_{\mathrm{F}}$ le domaine occupé par la cellule élémentaire. $\boldsymbol{u}_{\mathrm{S}}$ représente le déplacement de l'inclusion, $\boldsymbol{u}_{\mathrm{F}}$ le champ de déplacement du fluide (description locale) et $\boldsymbol{U}_{\mathrm{F}}$ le champ de déplacement moyen du fluide sur la cellule (description globale), défini comme la valeur moyenne du déplacement (solide et fluide) sur la cellule élémentaire, c'est-à-dire :

$$
\boldsymbol{U}_{\mathrm{F}}=\frac{\left|\Omega_{\mathrm{S}}\right|}{\left|\Omega_{\mathrm{T}}\right|} \boldsymbol{u}_{\mathrm{S}}+\frac{1}{\left|\Omega_{\mathrm{T}}\right|} \int_{\Omega_{\mathrm{F}}} \boldsymbol{u}_{\mathrm{F}} \mathrm{d} \Omega_{\mathrm{F}}
$$

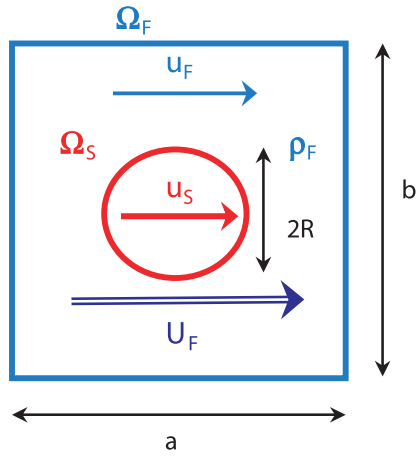

Fig. 4. Cellule élémentaire.

En utilisant les notations du paragraphe précédent, on identifie ainsi $\boldsymbol{u}_{\mathrm{S}} \equiv \boldsymbol{u}_{\mathrm{o}}, \dot{\boldsymbol{u}}_{\mathrm{F}} \equiv \boldsymbol{v}_{\mathrm{o}}$ et $\dot{\boldsymbol{U}}_{\mathrm{F}} \equiv \tilde{\boldsymbol{v}}_{\mathrm{o}}$. Sur le domaine fluide homogénéisé le champ de pression $p$ (identifié comme $p \equiv p_{\mathrm{o}}$ ) est calculé à partir du champ d'accélération moyen du fluide et de l'accélération de l'inclusion, grâce à une relation déduite de l'équation (6), écrite ainsi :

$$
\rho_{\mathrm{F}} \ddot{\boldsymbol{U}}_{\mathrm{F}}=-(1-J) \nabla p+\rho_{\mathrm{F}} J \ddot{\boldsymbol{u}}_{\mathrm{S}}
$$

Le ratio de confinement est tel que $J \in[0,1]$. Il est alors intéressant d'examiner les cas limites $J=0$ et $J=1$, qui correspondent aux situations suivantes :

- $J=0$ lorsque la cellule fluide ne contient pas d'inclusion solide. Dans ce cas, l'équation (8) donne la relation classique $\rho_{\mathrm{F}} \ddot{\boldsymbol{U}}_{\mathrm{F}}=\nabla p$.

- $J=1$ lorsque la cellule $\Omega_{\mathrm{T}}$ ne contient pas de fluide, l'équation (8) s'écrivant ainsi simplement $\ddot{\boldsymbol{U}}_{\mathrm{F}}=\ddot{\boldsymbol{u}}_{\mathrm{S}}$ : la cellule élémentaire est une cellule solide.

Le premier terme du membre de droite dans l'équation (8) exprime une correction du gradient de pression $\nabla p$ par le coefficient $1-J$ pour tenir compte de la présence des inclusions solides dans la cellule fluide. L'équation (8) traduit ainsi que pour un mouvement donné du fluide, le gradient de pression est augmenté par le confinement.

On combine l'équation de conservation de la masse écrite en moyenne sur la cellule élémentaire (Éq. (4) spécialisée au cas des inclusions rigides) avec l'équation d'état du fluide homogénéisé pour écrire par ailleurs :

$$
p=-\rho_{\mathrm{F}} c^{2} \nabla \cdot \boldsymbol{U}_{\mathrm{F}}
$$

\subsection{Problème couplé fluide/structure avec fluide homogénéisé}

Le modèle fluide homogénéisé avec inclusions solides peut être maintenant utilisé pour décrire l'interaction fluide/structure pour le fluide couplant les structures intérieures et extérieures du réacteur. La modélisation 


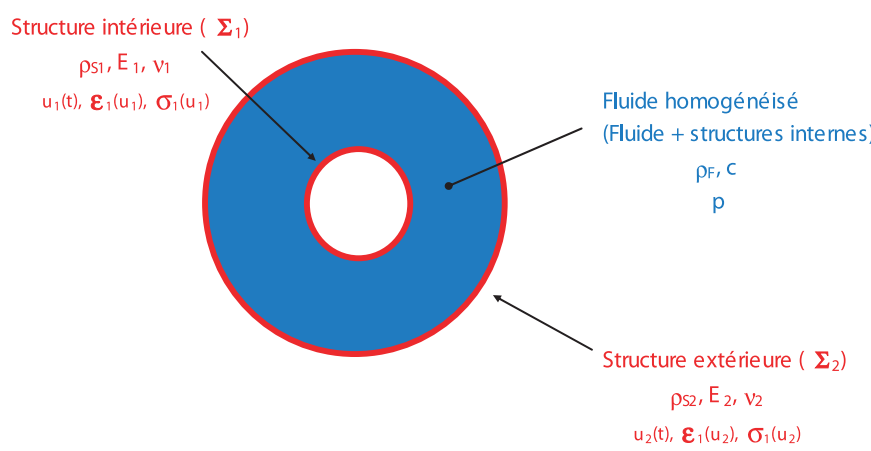

Fig. 5. Problème couplé fluide/structure avec fluide homogénéisé.

adoptée ici suppose que les mouvements du fluide se produisent de façon prépondérante dans un plan perpendiculaire à l'axe vertical du réacteur, cette hypothèse étant validée par le caractère relativement élancé de la structure. On rappelle en outre que les internes du réacteur sont solidaires de la structure de supportage et sont donc supposés liés rigidement à la coque interne.

Les équations du problème couplé structures/fluide homogénéisé sont alors écrites de la façon suivante. En considérant une tranche bidimensionnelle du problème, telle que représentée par la figure 5 , on note $\Sigma_{k}(k=1,2)$ les structures intérieures et extérieures du réacteur, de frontière $\partial \Sigma_{k}(k=1,2)$ avec $\partial \Sigma_{k}=\partial \Sigma_{k o} \cup \partial \Sigma_{k \sigma} \cup \Gamma_{k}$, où $\partial \Sigma_{k o}$ représente la frontière à déplacement contraint $\partial \Sigma_{k \sigma}$ la frontière libre d'efforts et $\Gamma_{k}$ la frontière en interface avec le fluide. Sur $\partial \Sigma_{k}$, la normale extérieure est notée $\boldsymbol{n}_{k}$. L'inconnue du problème structure est alors le champ de déplacement $\operatorname{sur} \Sigma_{1}$ et $\Sigma_{2}$, soit $\boldsymbol{u}_{1}$ and $\boldsymbol{u}_{2}$, respectivement. $\Omega$ représente par ailleurs le domaine fluide homogénéisé, de frontière $\partial \Omega$, avec $\partial \Omega=\partial \Omega_{\mathrm{o}} \cup \partial \Omega_{\pi} \cup \Gamma$, où $\partial \Omega_{\mathrm{o}}$ est la frontière à pression imposée, $\partial \Omega_{\pi}$ la frontière à gradient de pression imposé et $\Gamma$ la frontière en interface avec la structure. L'inconnue du problème fluide est le champ de pression $p$ (variable fluide à l'échelle globale $\operatorname{sur} \Omega$ ).

L'équation locale du problème fluide est obtenue à partir des équations (13) et (14) et s'écrit :

$$
\frac{\ddot{p}}{c^{2}}-(1-J) \Delta p=0
$$

avec les conditions aux limites :

$$
p=0 \quad \text { sur } \quad \partial \Omega_{\mathrm{o}} \quad \text { et } \quad \frac{\partial p}{\partial n}=0 \quad \text { sur } \quad \partial \Omega_{\mathrm{n}}
$$

et la condition de couplage avec les structures donnée par :

$\frac{\partial p}{\partial n}=-\rho_{\mathrm{F}} \ddot{\boldsymbol{u}} \cdot \boldsymbol{n} \quad$ (ou de façon équivalente

$$
\left.\boldsymbol{U}_{\mathrm{F}} \cdot \boldsymbol{n}=\boldsymbol{u} \cdot \boldsymbol{n}\right) \quad \text { sur } \quad \Gamma
$$

Les inclusions solides étant rigidement liées à la coque interne, on a de plus $\boldsymbol{u}_{\mathrm{S}} \equiv \boldsymbol{u}_{1}$ sur $\Gamma_{1}$. La formulation variationnelle du problème fluide s'écrit alors sous la forme suivante :

$$
\begin{array}{r}
\int_{\Omega} \frac{p \delta p}{c^{2}} \mathrm{~d} \Omega+\int_{\Omega}(1-J) \nabla p \cdot \nabla \delta p \mathrm{~d} \Omega=\int_{\Gamma_{1}}-\rho(1-J) \ddot{\boldsymbol{u}}_{1} \cdot \boldsymbol{n} \delta p \mathrm{~d} \Gamma \\
\quad+\int_{\Gamma_{2}}-\rho(1-J) \ddot{\boldsymbol{u}}_{2} \cdot \boldsymbol{n} \delta p \mathrm{~d} \Gamma+\int_{\Gamma_{2}}-\rho J \ddot{\boldsymbol{u}}_{1} \cdot \boldsymbol{n} \delta p \mathrm{~d} \Gamma
\end{array}
$$

Les équations locales du problème structure sont celles de l'élasticité linéaire pour chaque système $\Sigma_{k}$ :

$$
\rho_{\mathrm{S}, \mathrm{k}} \ddot{u}_{\mathrm{i}}^{k}-\frac{\partial \sigma_{\mathrm{ij}}\left(\boldsymbol{u}_{k}\right)}{\partial x_{\mathrm{j}}}=0
$$

avec les conditions aux limites :

$$
u_{\mathrm{i}}^{k}=0 \quad \text { sur } \quad \partial \Sigma_{\mathrm{ko}} \quad \text { et } \quad \sigma_{\mathrm{ij}}\left(\boldsymbol{u}_{k}\right) n_{j}^{k}=0 \quad \text { sur } \quad \partial \Sigma_{k \sigma}
$$

et la condition de couplage avec le fluide :

$$
\sigma_{\mathrm{ij}}\left(\boldsymbol{u}_{\mathrm{k}}\right) n_{\mathrm{j}}^{k}=p n_{\mathrm{i}} \quad \text { sur } \quad \Gamma_{\mathrm{k}}
$$

L'équation de la dynamique de la structure intérieure $\Sigma_{1}$ doit en outre prendre en compte les efforts exercés par le fluide sur les inclusions qui lui sont solidaires. Du point de vue local, il s'agit donc d'écrire les efforts fluides sur $\Omega_{\mathrm{S}}$, dont l'effet se traduit au niveau global par une densité d'effort d $\phi$ donnée par [19] :

$$
\mathrm{d} \phi=\left(-J \nabla p-\rho_{\mathrm{F}} I \frac{\left|\Omega_{\mathrm{F}}\right|}{\left|\Omega_{\mathrm{T}}\right|} \ddot{\boldsymbol{u}}_{\mathrm{S}}\right) \mathrm{d} \Omega
$$

avec $J=\frac{\left|\Omega_{\mathrm{S}}\right|}{\left|\Omega_{\mathrm{T}}\right|}+I \frac{\left|\Omega_{\mathrm{F}}\right|}{\left|\Omega_{\mathrm{T}}\right|}$.

L'équation (17) peut ainsi être vue comme l'équation duale de l'équation (8) pour le problème structure. Le travail virtuel de la force fluide $\phi$ dans le champ de déplacement virtuel $\delta \boldsymbol{u}_{1}$ pour la structure intérieure est alors calculé comme :

$$
\begin{aligned}
& \phi \cdot \delta \boldsymbol{u}_{1}=\left(\int_{\Omega} \mathrm{d} \boldsymbol{\phi}\right) \cdot \delta \boldsymbol{u}_{1}=-\int_{\Omega} \rho_{\mathrm{F}} I \frac{\left|\Omega_{\mathrm{F}}\right|}{\left|\Omega_{\mathrm{T}}\right|} \mathrm{d} \Omega \times \ddot{\boldsymbol{u}}_{1} \cdot \delta \boldsymbol{u}_{1} \\
& +\int_{\Gamma_{1}}-J p \boldsymbol{n} \cdot \delta \boldsymbol{u}_{1} \mathrm{~d} \Gamma_{1}+\int_{\Gamma_{2}}-J p \boldsymbol{n} \cdot \delta \boldsymbol{u}_{1} \mathrm{~d} \Gamma_{2}
\end{aligned}
$$

de sorte que la formulation variationnelle du problème structure s'écrive :

$$
\begin{aligned}
& \int_{\Sigma_{k}} \rho_{S, k} \ddot{u}_{\mathrm{i}}^{k} \delta u_{\mathrm{i}}^{k} \mathrm{~d} \Sigma_{\mathrm{k}}+\int_{\Sigma} \sigma_{\mathrm{ij}}\left(\boldsymbol{u}_{\mathrm{k}}\right) \varepsilon_{\mathrm{ij}}\left(\delta \boldsymbol{u}_{k}\right) \mathrm{d} \Sigma_{\mathrm{k}}= \\
& \int_{\Gamma_{1}} p(1-J) \boldsymbol{n} \cdot \delta \boldsymbol{u}_{1} \mathrm{~d} \Gamma_{1}+\int_{\Gamma_{2}} p \boldsymbol{n} \cdot \delta \boldsymbol{u}_{2} \mathrm{~d} \Gamma_{2}-\tilde{m}_{1} \ddot{\boldsymbol{u}}_{1} \cdot \delta \boldsymbol{u}_{1} \\
& +\int_{\Gamma_{2}}-J p \boldsymbol{n} \cdot \delta \boldsymbol{u}_{1} \mathrm{~d} \Gamma_{2}
\end{aligned}
$$

avec $\tilde{m}_{1}=\int_{\Omega} \rho_{\mathrm{F}} I \frac{\left|\Omega_{\mathrm{F}}\right|}{\left|\Omega_{\mathrm{T}}\right|} \mathrm{d} \Omega$. 


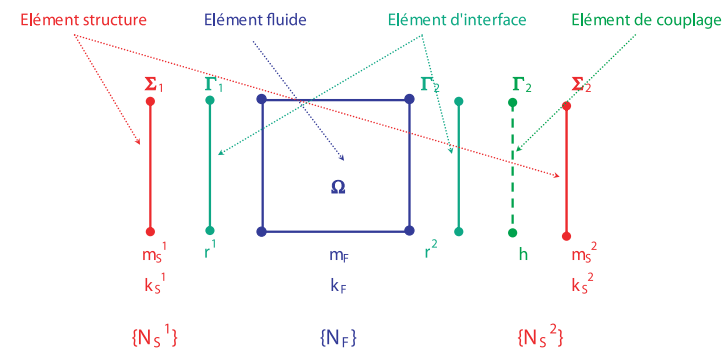

Fig. 6. Discrétisation éléments-finis : éléments-finis structure et fluide, éléments d'interface et de couplage.

\subsection{Discrétisation éléments-finis}

La discrétisation des équations (13) et (19) est conduite avec une technique éléments-finis, utilisant des éléments-finis structure, fluide et des éléments d'interface et de couplage (voir Fig. 6), permettant le calcul des opérateurs des problèmes structure et fluide et des opérateurs de couplage.

On obtient ainsi l'équation matricielle suivante :

$$
\begin{aligned}
& {\left[\begin{array}{lll}
\boldsymbol{M}_{\mathrm{S}}^{1}+\tilde{\boldsymbol{M}}_{1} & \mathbf{0} & \mathbf{0} \\
\mathbf{0} & \boldsymbol{M}_{\mathrm{S}}^{2} & \mathbf{0} \\
\rho_{\mathrm{F}}(1-J) \boldsymbol{R}_{1}^{T}-J \boldsymbol{H}^{T} & \rho_{\mathrm{F}} \boldsymbol{R}_{2}^{T} & \boldsymbol{M}_{\mathrm{F}}
\end{array}\right]\left\{\begin{array}{l}
\ddot{\boldsymbol{U}}_{1}(t) \\
\ddot{\boldsymbol{U}}_{2}(t) \\
\ddot{\boldsymbol{P}}(t)
\end{array}\right\}} \\
& +\left[\begin{array}{lll}
\boldsymbol{K}_{\mathrm{S}}^{1} & \mathbf{0} & -(1-J) \boldsymbol{R}_{1}+J \boldsymbol{H} \\
\mathbf{0} & \boldsymbol{K}_{\mathrm{S}}^{2} & \boldsymbol{R}_{2} \\
\mathbf{0} & \mathbf{0} & (1-J) \boldsymbol{K}_{\mathrm{F}}
\end{array}\right]\left\{\begin{array}{c}
\boldsymbol{U}_{1}(t) \\
\boldsymbol{U}_{2}(t) \\
\boldsymbol{P}(t)
\end{array}\right\}=\left\{\begin{array}{l}
\mathbf{0} \\
\mathbf{0} \\
\mathbf{0}
\end{array}\right\}
\end{aligned}
$$

La discrétisation des termes de couplage sur chaque interface fluide/structure $\Gamma_{\mathrm{k}}$ est réalisée avec des éléments d'interface et permet d'obtenir les opérateurs d'interaction $\boldsymbol{R}_{\mathrm{k}}$ :

$$
\int_{\Gamma_{k}} p \boldsymbol{n} \cdot \delta \boldsymbol{u}_{k} \mathrm{~d} \Gamma \rightarrow \delta \boldsymbol{U}_{k}^{\mathrm{T}} \boldsymbol{R}_{k} \boldsymbol{P}
$$

Le couplage des structures intérieure et extérieure par les inclusions solides est décrit au moyen de l'opérateur de couplage $\boldsymbol{H}$ défini comme :

$$
\int_{\Gamma_{2}} p \boldsymbol{n} \cdot \delta \boldsymbol{u}_{1} \mathrm{~d} \Gamma \rightarrow \delta \boldsymbol{U}_{1} \boldsymbol{H} \boldsymbol{P}
$$

Les efforts exercés par le fluide sur la structure intérieure $\Sigma_{1}$ par les internes sont décrits au moyen d'un opérateur de masse ajoutée $\tilde{M}_{1}$, défini comme :

$$
\int_{\Omega} \rho_{\mathrm{F}} I \frac{\left|\Omega_{\mathrm{F}}\right|}{\left|\Omega_{\mathrm{T}}\right|} \mathrm{d} \Omega \times \ddot{\boldsymbol{u}}_{1} \cdot \delta \boldsymbol{u}_{1} \rightarrow \delta \boldsymbol{U}_{1}^{T} \tilde{\boldsymbol{M}}_{1} \boldsymbol{U}_{1}
$$

Les matrices de masse et de raideur apparaissant dans la formulation (20) sont non symétriques, ce qui est un résultat classique en formulation pression/ déplacement [5]. Le calcul des modes propres et des fréquences propres peut être réalisé en utilisant des algorithmes de recherche de valeurs propres adaptés au cas non symétrique [20]. Les modes propres ainsi obtenus ne vérifient cependant pas les conditions d'orthogonalité requises pour la mise en ouvre de méthodes par projection sur base modale. L'utilisation d'une formulation couplée basée sur la description du problème avec les variables $(\boldsymbol{u}, p, \varphi)[3]$ permettra alors de travailler avec des opérateurs de masse et de raideur symétriques, utilisant les opérateurs élémentaires apparaissant dans la formulation (20).

\subsection{Calcul du ratio de confinement}

Le ratio de confinement $J$ peut être calculé théoriquement à partir de l'équation (6) en évaluant $\Pi_{\mathrm{i}}$ sur une cellule élémentaire (voir Fig. 7), en utilisant si nécessaire une méthode numérique appropriée. Il est cependant possible de proposer une approche plus directe [15]. Les efforts de pression agissant sur une inclusion solide $\Omega_{\mathrm{S}}$ en mouvement dans une cellule fluide $\Omega_{\mathrm{F}}$ (voir Fig. 4) se calculent en effet comme :

$$
\mathbf{\Phi}=-m_{\mathrm{h}} \ddot{\boldsymbol{u}}_{\mathrm{S}}+\left(m_{\mathrm{h}}+m_{\mathrm{d}}\right) \ddot{\boldsymbol{U}}_{\mathrm{F}}
$$

où $m_{\mathrm{h}}$ est la masse de fluide ajoutée sur l'inclusion et $m_{\mathrm{d}}=\rho_{\mathrm{F}}\left|\Omega_{\mathrm{S}}\right|$ est la masse de fluide déplacée par l'inclusion. Les forces exercées sur le fluide s'expriment par ailleurs comme $-\left|\Omega_{\mathrm{T}}\right| \nabla p-\boldsymbol{\Phi}$ [19], de sorte qu'on peut écrire :

$$
\int_{\Omega_{\mathrm{F}}} \rho_{\mathrm{F}} \ddot{\boldsymbol{u}}_{\mathrm{F}} \mathrm{d} \Omega_{\mathrm{F}}=-\left|\Omega_{\mathrm{T}}\right| \nabla p+m_{\mathrm{h}} \ddot{\boldsymbol{u}}_{\mathrm{S}}-\left(m_{\mathrm{h}}+\rho_{\mathrm{F}}\left|\Omega_{\mathrm{S}}\right|\right) \ddot{\boldsymbol{U}}_{\mathrm{F}}
$$

En tenant compte de l'équation (7), on déduit :

$$
\begin{gathered}
\left(\rho_{\mathrm{F}}\left(\left|\Omega_{\mathrm{T}}\right|+\left|\Omega_{\mathrm{S}}\right|\right)+m_{\mathrm{h}}\right) \ddot{\boldsymbol{U}}_{\mathrm{F}}= \\
-\left|\Omega_{\mathrm{T}}\right| \nabla p+\left(m_{\mathrm{h}}+\rho_{\mathrm{F}}\left|\Omega_{\mathrm{S}}\right|\right) \ddot{\boldsymbol{u}}_{\mathrm{S}}
\end{gathered}
$$

d'où :

$$
\rho_{\mathrm{F}} \ddot{\boldsymbol{U}}_{\mathrm{F}}=-(1-J) \nabla p+J \rho_{\mathrm{F}} \ddot{\boldsymbol{u}}_{\mathrm{S}}
$$

ce qui est l'équation (8), avec $J$ donné par :

$$
J=\left(m_{\mathrm{h}}+\rho_{\mathrm{F}}\left|\Omega_{\mathrm{S}}\right|\right) /\left(m_{\mathrm{h}}+\rho_{\mathrm{F}}\left(\left|\Omega_{\mathrm{T}}\right|+\left|\Omega_{\mathrm{S}}\right|\right)\right)
$$

L'expression (28) est une définition alternative de $J$ qui permet son calcul effectif en utilisant un modèle élémentaire de la cellule fluide pour évaluer le terme de masse ajoutée. Ce dernier est ainsi déterminé en supposant que l'inclusion rigide est un système masse/rigidité $m, k$ élémentaire. En comparant la pulsation dans le vide $\Omega_{\mathrm{o}}=\sqrt{\frac{k}{m}}$ avec la pulsation dans le fluide $\Omega_{\mathrm{h}}=\sqrt{\frac{k}{m+m_{\mathrm{h}}}}$ (déterminée avec un calcul sur la cellule élémentaire, Fig. 7), on déduit $m_{\mathrm{h}}$ puis le ratio de confinement $J$ en appliquant l'équation (28).

\subsection{Calcul des masses effectives}

La méthode proposée ici est appliquée à l'analyse du comportement au séisme du réacteur, décrit par l'équation dynamique :

$$
\boldsymbol{M} \ddot{\boldsymbol{X}}(t)+\boldsymbol{K} \boldsymbol{X}(t)=-\boldsymbol{M} \Delta \gamma(t)
$$




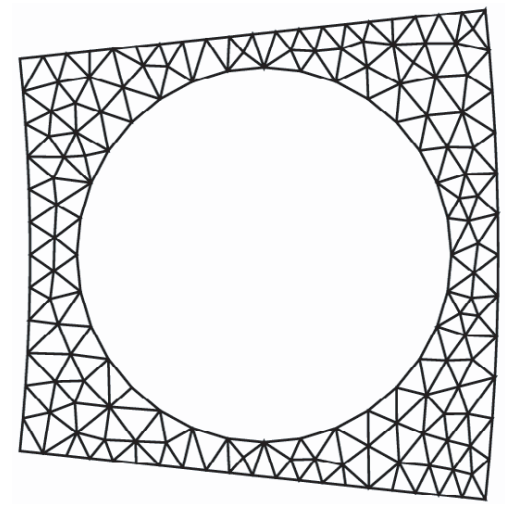

Fig. 7. Cellule fluide élémentaire.

où $\boldsymbol{M}$ et $\boldsymbol{K}$ sont les opérateurs de masse et de raideur donnés par l'équation (20). $\boldsymbol{\Delta}$ représente la direction de l'accélération $\gamma(t)$ décrivant le séisme. L'équation (29) peut être intégrée en utilisant une technique de projection sur base modale. Les modes propres $\left(\boldsymbol{X}_{n}\right)_{n \geqslant 1}$ et les pulsations propres $\left(\Omega_{n}\right)_{n \geqslant 1}$ sont obtenus par résolution du problème aux valeurs propres $\left(\boldsymbol{K}-\Omega_{n}^{2} \boldsymbol{M}\right) \boldsymbol{X}_{n}=0$. La contribution de chaque mode dans la réponse d'ensemble du système est quantifiée par la masse effective $\mu_{n}$ du mode, définie par :

$$
\mu_{\mathrm{n}}=\frac{\left(\boldsymbol{\Delta}^{T} \boldsymbol{M} \boldsymbol{X}_{\mathrm{n}}\right)^{2}}{\boldsymbol{X}_{\mathrm{n}}^{T} \boldsymbol{M} \boldsymbol{X}_{\mathrm{n}}}
$$

La somme des masses effectives donne la masse totale $m$ (structure et fluide) du système soumis à la sollicitation dynamique :

$$
\sum_{\mathrm{n} \geqslant 1} \mu_{\mathrm{n}}=\boldsymbol{\Delta}^{\mathrm{T}} \boldsymbol{M} \Delta=m
$$

On montre alors que la méthode d'homogénéisation proposée ici vérifie cette dernière équation [21] et qu'elle est ainsi consistante avec une description de la dynamique sous séisme du système étudié (calcul des modes propres, des fréquences propres et des masses effectives).

\section{Validation et application de la méthode}

\subsection{Validation par comparaison entre calculs 3D et calculs $2 \mathrm{D}$}

La méthode d'homogénéisation proposée est validée par comparaison entre des calculs de modes, fréquences et masses effectives du réacteur avec un modèle tridimensionnel (modélisation complète des internes du réacteur et de l'espace fluide) et un modèle bidimensionnel axi-symétrique équivalent (modélisation avec internes et fluide homogénéisés), voir figure 8.

Dans l'approche 2D axi-symétrique, on utilise un développement en série de Fourier des inconnues du problème (déplacement structure $\boldsymbol{u}$, pression et potentiel

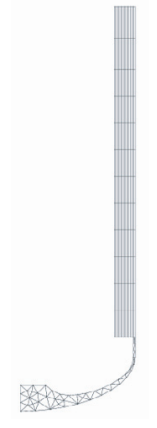

Modèle 2D
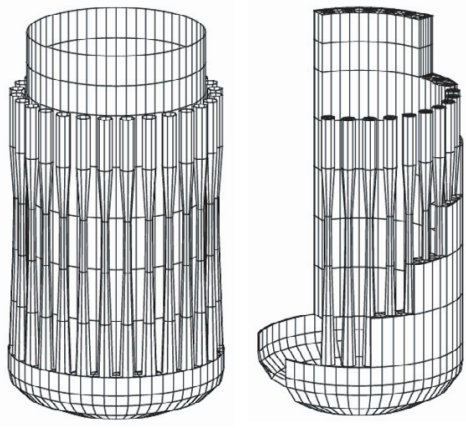

Modèle 3D
Fig. 8. Modèle $3 \mathrm{D}$ et $2 \mathrm{D}$ axi-symétrique du réacteur.
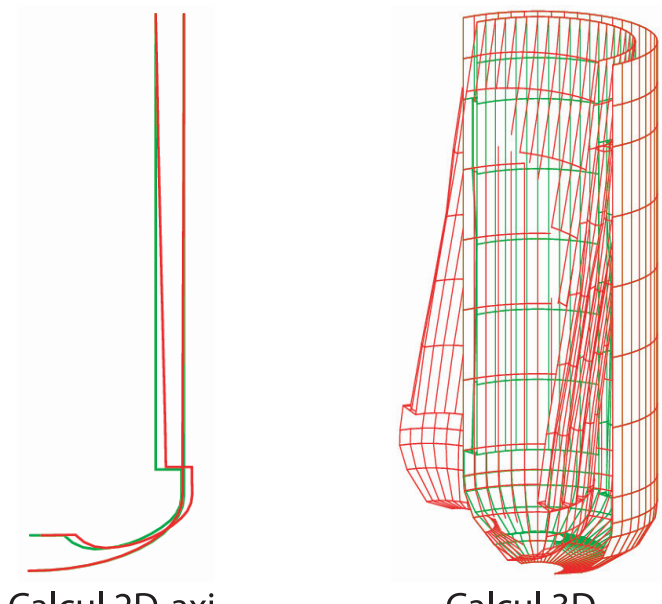

Calcul 3D

Fig. 9. Déformée modale pour le premier mode de la structure sèche.

des déplacements fluide $p, \varphi)$ :

$\left\{\begin{array}{l}u_{r}(r, \theta, z) \\ u_{\theta}(r, \theta, z) \\ u_{z}(r, \theta, z) \\ p(r, \theta, z) \\ \varphi(r, \theta, z)\end{array}\right\}=\left\{\begin{array}{l}u_{r}^{0}(r, z) \\ u_{\theta}^{0}(r, z) \\ u_{z}^{0}(r, z) \\ p^{0}(r, z) \\ \varphi(r, \theta, z)\end{array}\right\}$

$+\sum_{s \geqslant 1}\left\{\begin{array}{l}u_{r}^{s}(r, z) \\ u_{\theta}^{s}(r, z) \\ u_{z}^{s}(r, z) \\ p^{s}(r, z) \\ \varphi^{s}(r, \theta, z)\end{array}\right\} \cdot \cos (s \theta)+\sum_{a \geqslant 1}\left\{\begin{array}{l}u_{r}^{a}(r, z) \\ -u_{\theta}^{a}(r, z) \\ u_{z}^{a}(r, z) \\ p^{a}(r, z) \\ \varphi^{a}(r, \theta, z)\end{array}\right\} \cdot \sin (a \theta)$

Les modes sismiques sont les modes de Fourier symétriques et antisymétriques d'ordre un $(s=1$ et $a=1$ ). Les calculs éléments finis sont réalisés avec le code CASTEM. En négligeant la raideur des internes, on vérifie que les calculs $2 \mathrm{D}$-axi et $3 \mathrm{D}$ donnent des résultats identiques pour la structure sèche, que ce soit en terme de déformées modales, fréquences propres et masses effectives (Fig. 9 et Tab. 1).

Dans le cas couplé, le calcul 2D-axi avec fluide homogénéisé est réalisé en divisant l'espace fluide en dix zones suivant la direction verticale (de la partie inférieure 
Tableau 1. Modes propres de la structure sèche, calculs 2D et 3D.

\begin{tabular}{|c|c|c|c|c|}
\hline \multirow{2}{*}{ Mode } & Modèle 2D-axi & \multicolumn{2}{|c|}{ Modèle 3D } & \multirow[b]{2}{*}{$\mu_{n}[\%]$} \\
\hline & $f_{n}[\mathrm{~Hz}]$ & $\mu_{n}[\%]$ & $f_{n}[\mathrm{~Hz}]$ & \\
\hline 1 & 43,8 & 39,83 & 44,5 & 39,60 \\
\hline 2 & 81,3 & 28,61 & 82,9 & 28,41 \\
\hline 3 & 138,3 & 11,38 & 139,3 & 11,36 \\
\hline
\end{tabular}

Tableau 2. Calcul du coefficient de confinement pour les différentes zones du réacteur.

\begin{tabular}{cc}
\hline Zone \# & Confinement coefficient $J$ \\
\hline 1 & 0,57 \\
2 & 0,45 \\
3 & 0,34 \\
4 & 0,24 \\
5 & 0,15 \\
6 & 0,13 \\
7 & 0,23 \\
8 & 0,62 \\
9 & 0,62 \\
10 & 0,44 \\
\hline
\end{tabular}

Tableau 3. Modes propres du réacteur avec interaction fluide/structure : calcul 2D-axisymétrique avec fluide homogénéisé et calcul 3D avec modélisation de internes.

\begin{tabular}{ccccc}
\hline \multirow{2}{*}{ Mode } & \multicolumn{2}{c}{ Modèle 2D-axi } & \multicolumn{2}{c}{ Modèle 3D } \\
\cline { 2 - 5 } & $f_{n}[\mathrm{~Hz}]$ & $\mu_{n}[\%]$ & $f_{n}[\mathrm{~Hz}]$ & $\mu_{n}[\%]$ \\
\hline 1 & 21,291 & 2,80 & 21,302 & 2,79 \\
2 & 64,750 & 70,1 & 64,761 & 70,0 \\
3 & 82,722 & 1,48 & 82,732 & 1,45 \\
4 & 88,807 & 4,01 & 88,964 & 4,05 \\
5 & 163,37 & 2,88 & 163,74 & 2,72 \\
\hline
\end{tabular}

à la partie supérieure du réacteur), pour lesquelles un coefficient de confinement est calculé (Tab. 2) [22].

Les résultats de calculs $2 \mathrm{D}$-axi et $3 \mathrm{D}$ avec couplage fluide/structure sont comparés dans le tableau 3 (qui donne les fréquences et masses effectives des cinq premiers modes) et à la figure 10 (qui représente les déformées modales des deux premiers modes).

Les deux calculs donnent des résultats similaires en terme de déformées modales (déplacement structure, pression fluide), de fréquences propres et de masse modale (rapporté à la masse totale du système) : le premier mode propre est un mode de flexion de la structure intérieure (à la fréquence de $21,3 \mathrm{~Hz}$ et représentant $2,80 \%$ de la masse totale), le second mode est un mode de flexion couplée des structures intérieure et extérieure (à la fréquence de $64,7 \mathrm{~Hz}$ et représentant 70,1\% de la masse totale). Le modèle $2 \mathrm{D}$ « simplifié » permet ainsi une description correcte des effets de couplage et identique à celle qui est prévue par le modèle $3 \mathrm{D}$ « complet » et permet de substituer à une modélisation tridimensionnelle, prenant en compte les détails de géométrie des structures internes du réacteur, une modélisation bidimensionnelle axisymétrique équivalente.

\subsection{Influence des structures internes : effet de confinement}

La méthode d'homogénéisation permet alors d'évaluer rapidement et simplement les effets de confinement induits par la présence des internes dans le réacteur. Le tableau 4 compare à titre d'illustration les résultats de calculs couplés avec et sans prise en compte des internes.

Les fréquences propres couplées, calculées avec modélisation des internes sont inférieures aux fréquences calculées sans modélisation des internes, ce qui illustre clairement l'effet de confinement induit par la présence des structures internes du réacteur, qui tendent à limiter les mouvements du fluide et à accroître ainsi les effets de couplage inertiels. Pour le premier mode propre (flexion d'ensemble de la structure intérieure), la baisse en fréquence est assez significative $(-12 \%)$; elle est plus importante pour le second mode (flexion couplée en phase des structures intérieure et extérieure), de l'ordre de $-16 \%$. Pour le troisième mode (flexion couplée en opposition de phase des structures intérieure et extérieure), l'influence est du même ordre de grandeur que pour le premier mode.

\section{Conclusion}

Une méthode d'homogénéisation permettant la modélisation des structures internes d'un réacteur nucléaire et la description des effets d'interaction fluide/structure a été présentée dans cet article. Les principes théoriques de base de la méthode ont été rappelés et une validation de la méthode a été proposée. En particulier, il a été montré que la méthode permet un calcul des modes propres et des masses effectives du réacteur tenant compte des effets de couplage inertiels et de confinement.

La validation numérique de la méthode a été établie par comparaison entre une modélisation tridimensionnelle « complète » du réacteur avec détail de la géométrie des structures internes et des espaces fluides et une modélisation bidimensionnelle « simplifiée » utilisant un fluide homogène équivalent. Il a été montré que les modèles $2 \mathrm{D}$ et $3 \mathrm{D}$ donnent des résultats identiques, à la fois en terme de calcul de fréquences propres, déformées modales et masses effectives.

Du point de vue industriel, les principales conclusions de cette étude sont les suivantes :

(i) la méthode d'homogénéisation proposée constitue une approche numérique simple et efficace qui permet de substituer à une modélisation tridimensionnelle prenant en compte les détails de géométrie des structures internes du réacteur une modélisation bidimensionnelle axisymétrique équivalente : les détails de la géométrie des internes du réacteur sont pris en compte à l'échelle globale l'aide d'un coefficient scalaire, calculé sur une cellule élémentaire, représentative de la géométrie à l'échelle locale;

(ii) l'implémentation de la méthode dans un code éléments-finis est immédiate, et nécessite le calcul 

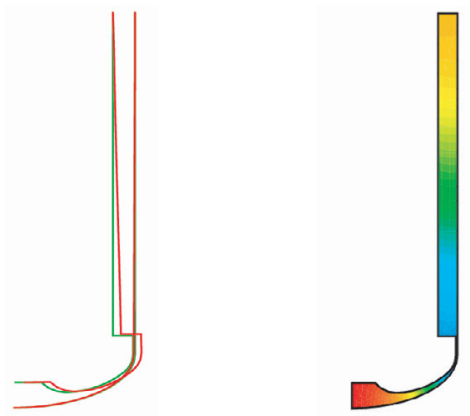

Calcul 2D-axi-f ${ }_{1}=21.3 \mathrm{~Hz}, \mu_{1}=2.80 \%$
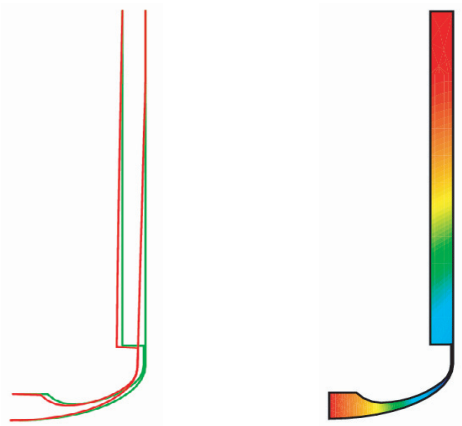

Calcul 2D-axi- $f_{2}=64.7 \mathrm{~Hz}, \mu_{2}=70.1 \%$
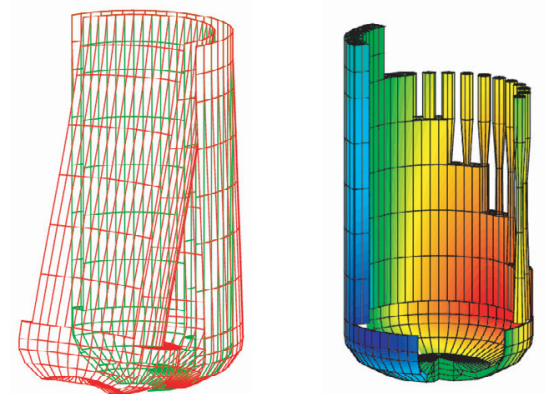

Calcul 3D $-\mathrm{f}_{1}=21.3 \mathrm{~Hz}, \mu_{1}=2.79 \%$
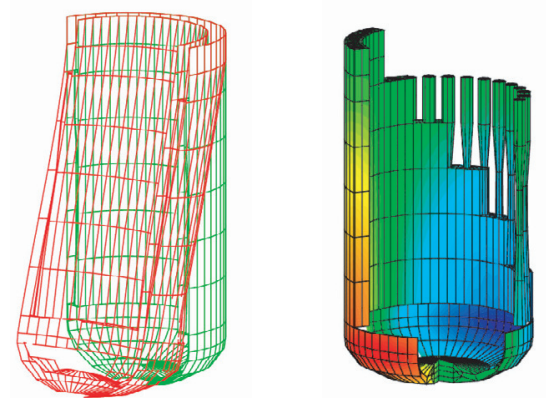

Calcul 3D $-\mathrm{f}_{2}=64.8 \mathrm{~Hz}, \mu_{2}=70.0 \%$

Fig. 10. Déformées modales calculées avec les modélisations 2D et 3D.

Tableau 4. Influence des structures internes du réacteur sur l'interaction fluide/structure : effet de confinement.

\begin{tabular}{ccc}
\hline Mode & Sans modélisation des internes & Avec modélisation des internes \\
\hline 1 & $24,51 \mathrm{~Hz}$ & $21,29 \mathrm{~Hz}$ \\
2 & $77,21 \mathrm{~Hz}$ & $64,75 \mathrm{~Hz}$ \\
3 & $98,38 \mathrm{~Hz}$ & $82,72 \mathrm{~Hz}$ \\
\hline
\end{tabular}

d'opérateurs de couplage déduits des opérateurs d'interaction fluide/structure « classiques »;

(iii) la présence des structures internes du réacteur est caractérisée par un effet de confinement qui tend à accroître les couplages inertiels entre les structures extérieure (enveloppe résistante) et intérieure (supportage de cour). En conséquence, les modes propres du système couplé sont caractérisés par des fréquences diminuées de $15 \%$ environ dans une modélisation prenant en compte les internes, par rapport à une modélisation approchée négligeant leur présence.

Remerciements. Les auteurs remercient M. Guillopé et M. Glavicic du STXN pour le soutien apporté aux échanges scientifiques entre le Laboratoire d'Étude de Mécanique Sismique du CEA Saclay et le Service Technique et Scientifique de DCN Propulsion autour des études de modélisation de l'interaction fluide/structure et leur application aux projets de réacteurs de propulsion navale.

\section{Références}

[1] R.J. Fritz, The Effect of Liquids on the Dynamic Motion of Immersed Solids, J. Engineering for Industry 94 (1972) $167-173$
[2] R.J. Gibert, Vibration des structures. Interactions avec les fluides. Sources d'excitation aléatoires, Collection de la Direction des Études et Recherches d'Électricité de France, 69, Eyrolles, 1986

[3] H.J.-P. Morand, R. Ohayon, Fluid Structure Interaction, Wiley \& Sons, 1995

[4] J. Makerle, Fluid-structure interaction problems, finite element approach and boundary elements approaches, A bibliography, Finite Elements in Analysis and Design 31 (1999) 231-240

[5] F. Axisa, Modelling of Mechanical Systems, Vol. 3 : FluidStructure Interaction, Elsevier, 2006

[6] J.F. Sigrist, C. Lainé, B. Peseux, Analyse modale d'une structure industrielle avec prise en compte du couplage fluide/structure, Mécanique \& Industries 6 (2005) 553563

[7] J.F. Sigrist, Analyse dynamique d'une structure industrielle avec prise en compte du couplage fluide/structure, Mécanique \& Industries 7 (2006) 453-464

[8] J.F. Sigrist, Modal Analysis of Fluid-Structure Interaction Problems with Pressure-based Finite Elements for Industrial Applications, Int. J. Multiphysic, in press

[9] A. Bensoussan, J.L. Lions, G. Papanicolaou, Asymptotic Analysis for Periodic Structures, North-Holland, 1978 
[10] E. Sanchez-Palencia, Non Homogenous Media and Vibration Theory, Springer-Verlag, 1980

[11] C. Conca, J. Planchard, M. Vanninathan, Fluids and Periodic Structures, Masson, 1995

[12] J. Planchard, Eigenfrequencies of a Tube Bundle Placed in a Confined Fluid, Computer Methods in Applied Mechanics and Engineering 30 (1982) 75-93

[13] D. Brochard, F. Gantenbein, R.J. Gibert, Homogenisation of Tube Bundle, Application to LMFBR Core Analysis, 9th Conference on Structural Mechanic in Reactor Technology, Lausanne, August 1987

[14] K. Cheval, Modélisation du comportement sismique de structures multitubulaires baignées par un fluide dense, Thèse de Doctorat, Université Paris VI, 2001

[15] D. Broc, J.C. Queval, E. Viallet, Seismic Behaviour of PWR Reactor Cores: Fluid-Structure Effects, 17th Conference on Structural Mechanic in Reactor Technology, Prague, August 2003

[16] D. Brochard, F. Gantenbein, R.J. Gibert, Modelling of the Dynamic Behaviour of LWR Internals, 9th Conference on Structural Mechanic in Reactor Technology, Lausanne, August 1987
[17] R.W. Clough, J. Penzien, Dynamics of Structures, McGraw-Hill, 1986

[18] L. Hammami, Étude de l'interaction fluide/structure dans les faisceaux de tubes par une méthode d'homogénéisation. Application à l'analyse sismique des cœurs RNR, Thèse de Doctorat, Université Paris VI, 1991

[19] J.F. Sigrist, D. Broc, Une méthode d'homogénéisation pour l'analyse modale d'un problème d'interaction fluide/structure, Revue Européenne de Mécanique Numérique 7-8 (2006) 867-889

[20] C. Rajakumar, C.G. Rogers, The Lanczos Algorithm Applied to Unsymmetric Generalized Eigenvalue Problem, Inter. J. Numerical Methods in Engineering 32 (1991) 1009-1026

[21] J.F. Sigrist, D. Broc, A Homogenization Method for the Modal Analysis of a Nuclear Reactor with FluidStructure Interaction, Pressure Vessel and Piping, Vancouver, 25-28 July 2006

[22] J.F. Sigrist, D. Broc, Fluid-Structure Interaction: Validation of a Homogenisation Method, Pressure Vessel and Piping, Vancouver, 25-28 July 2006 